\title{
Implementation of Distributed Mobile Storage and Location-based Message Sharing Among Smart Phones
}

\author{
Hiroyuki Kasai Reona Mogi \\ The University of Electro-Communications, Japan
}

\begin{abstract}
Our new distributed mobile cache system enables to cash data temporarily in a designated local are. This needs no infrastructure network and servers. That is why this is sure to accelerate new location-based communication services. This paper reports an implementation of a middleware of the cache system. Furthermore, an implemented location-based message sharing application is detailed that utilizes the implemented middleware via application programming interfaces.
\end{abstract}

\section{Keywords}

Distributed Mobile Storage, WLAN, Message Sharing, Smart Phone

\section{INTRODUCTION}

With the advance of mobile $\mathrm{CE}$ devices equipped with short-range wireless communication technologies such as IEEE801.11, new services among nearby passing people are growing rapidly. Timeoffset communication capability will engender a new service paradigm and promote new service emergence. We proposed before a new distributed mobile cache system to cache data temporarily in a designated local area [1]. This paper reports a prototype system of this mobile cache system and its application on it.

\section{Area-based Distributed Mobile Cache [1]}

The area-based mobile cache system is formed by distributed multiple terminals; it is maintained by collaboratively sharing and relaying cache data among these terminals that pass in/near the local area. Pre-placed storage servers or static mobile terminals in the target cache area and the Internet infrastructure are not necessary. Such a caching capability can be provided anywhere if mobile terminals exist. Additionally, the cache data are not propagated over other areas in a borderless fashion, which enables applications to leverage this cache system anywhere, for example, in crowded city areas or large exhibition centers. The central contribution of this paper was the new relay data algorithms that achieve lower overhead communication and more robust caching capability. Regarding managing a location or area in a real physical space, although GPS can be applied, we also leverage WLAN communication technology itself. More specifically, detectable WLAN AP information is used to recognize the area as a landmark. The concept is shown in Fig.1.

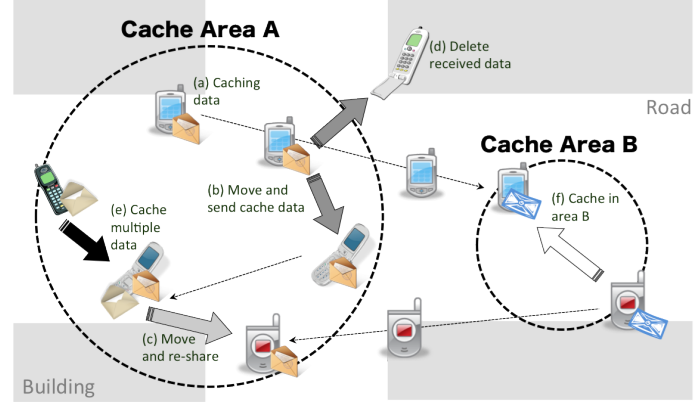

Fig. 1.Area-based Distributed Mobile Cache System [1].

\section{Message Sharing Application}

The implemented application enables users to share their messages within a designated public physical area. A user can post a message in a certain area, and the others who visit this area later can get it. This application looks like a local-area "Twitter" without Cloud server, or a kind of digital signage in a pubic area with no large display. Fig.2 shows a screen shot of the display mode of messages. If a terminal detects multiple WLAN APs, multiple sharing area indicated by sphere shape are shown on the display. The messages on the overlapped area between two spheres are those posted from the terminal that detects two APs at that time. Fig. 3 shows a screen shot for message posting. A new message posted into the area flashes in yellow to be noticed on the display. This application also can display various statistic data for the platform as depicted in Fig.4. This includes the number of relayed data, transmitted data, discarded data, detected APs, detected terminals in the ad-hoc communication, and its possessing messages.

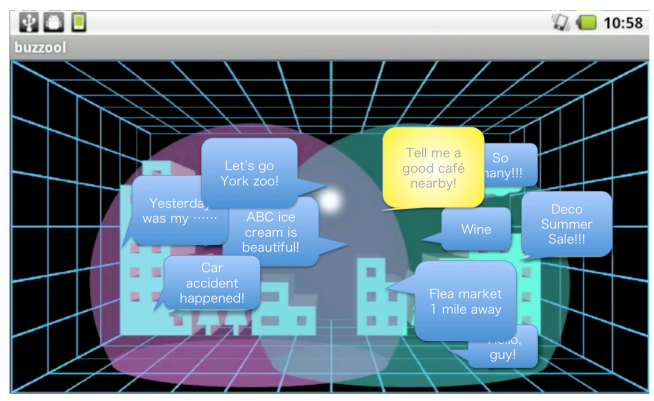

Fig.2. Message display interface.

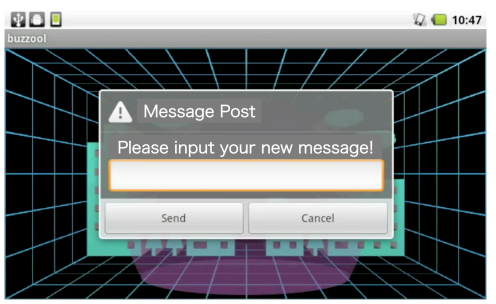

Fig.3. Message post interface.

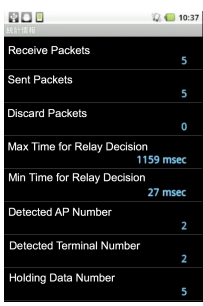

Fig.4. Statistics data.

\section{Conclusion}

This paper reported an implementation of a middleware of the cache system. In addition, an implemented location-based message sharing application is detailed that utilizes the middleware via application programming interfaces.

\section{REFERENCES}

[1] Hiromi Narimatsu, Hiroyuki Kasai and Ryoichi Shinkuma, "Area-based Collaborative Distributed Cache System using Consumer Electronics Mobile Device," IEEE Transaction on Consumer Electronics, Vol.57, No.2, pp. 564-573, 2011 